\title{
Treatment of the Ascending Aorta Aneurysm in a Patient with Horse Shoe Kidney
}

\author{
Serkan Burc Deser ${ }^{1^{*}}$
}

${ }^{1}$ Department of Cardiovascular Surgery, Medical Faculty, Ondokuz Mayis University, Samsun, Turkey

Correspondence:

Serkan Burc Deser

Address: Department of

Cardiovascular Surgery, Medical

Faculty, Ondokuz Mayis University, Samsun, Turkey

Email: sbd983@yahoo.com

Received: 14.07.2020,

Accepted: 22.09.2020

https://doi.org/10.29333/jcei/9561

\section{INTRODUCTION}

The Horse Shoe Kidney (HSK) is one of the most common congenital malformation of lower poles fusion of kidneys [1]. The reported incidence ranges from $0.15 \%$ to $0.33 \%$. Usually occurred during the $4-6$ th weeks of gestation. Mostly seen in males with 60-80\% vascular anomalies [2]. The coincidence of HSK and an ascending aortic aneurysm (AsAA) is rarely seen. To our knowledge, we report the first case of incidental HSK in a patient underwent Bentall procedure due to Ascending Aorta Aneurysm (AsAA).

\section{CASE REPORT}

A 65-year-old male was presented with dyspnea (New York Heart Association Functional Class II-III). He had a history of hypertension. His blood pressure was 140/80 $\mathrm{mmHg}$ and heart rate was $90 /$ minute. On physical examination, neither palpable mass nor pain was observed in the abdomen. Peripherical pulses were normal. Renal function and other laboratory tests were within normal limits. The Chest X-ray showed an enlarged AsAA. Computed tomography angiography (CTA) scanning showed an AsAA measuring $55 \mathrm{~mm}$ with no other aneurysms elsewhere and a coexistent HSK with a lower pole fusion (Figure 1a, 1b). The transthoracic echocardiography revealed $60 \%$ of left ventricular ejection fraction, mild mitral and severe aortic regurgitation. A coronary artery angiography was performed before the surgery and coronary arteries were found free of disease. Routine preparation for surgery was made and informed consent was taken. Median sternotomy and standard cannulation were performed. Subsequently, a single dose of antegrade isothermic blood cardioplegia was administered to arrest the heart. Cardiopulmonary bypass (CPB) was performed with moderate hypothermia. Ascending aorta, aortic root and aortic valve were replaced with 23 No valve (St. Jude Medical Inc. St Paul, MN, USA) and 28 No Dacron graft by performing Bentall procedure (Figure 1c,1d). The postoperative course was uneventful and he was discharged with oral anticoagulation. Six months later he was doing well with stable vital signs.

\section{DISCUSSION}

Ectopia, malrotation and vascular anomalies are usually seen in patients with HSK and are more prone to ascending urinary tract infections due to intestinal gram-negative organisms. $15-30 \%$ of patients with HSK are asymptomatic $[3,4]$. Despite, HSK is relatively seen with a high incidence of $0.15-0.33 \%$ in normal population, accompanying with abdominal aorta aneurysm is rarely seen with an incidence of $0.12 \%$ [5]. Unfortunately, there is no data for the incidence rate of association with AsAA. Besides that, the ascending aorta aneurysm is a lethal disease 

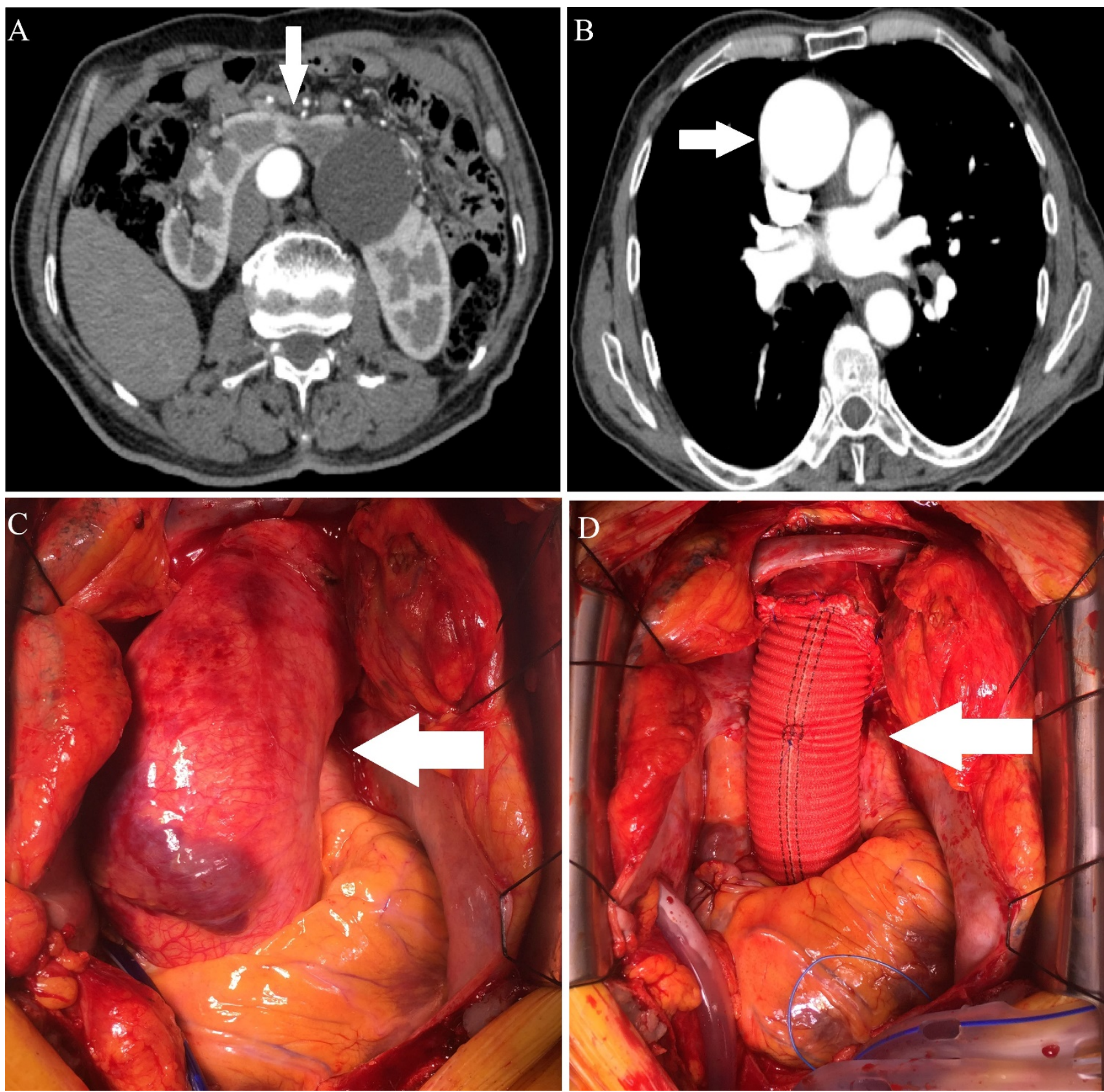

Figure 1. Preoperative two-dimensional computed tomography angiography (CTA) image of Horse Shoe Kidney (white arrow shows) (A) and Ascending Aorta Aneurysm (white arrow shows) (B). Perioperative external view of the Ascending Aorta Aneurysm (white arrow shows) (C), replacement ascending aorta and aortic root with a Dacron graft (white arrow shows) (D)

and usually found incidentally during transthoracic echocardiography. Aortic rupture and dissection are the potential complications due to weakened wall structure and hypertension. Surgical repair remains the gold standard treatment method in patients with a $>5.5 \mathrm{~cm}$ in diameter [6].

In conclusion, HSK is rarely seen with AsAA as it is in our case. The patient's history and Chest X-ray give us preliminary information. Clinicians should pay attention to the enlargement of the aortic shadow on Chest X-ray for possible ascending aorta aneurysm. Functional aortic valve insufficiency is a common condition in ascending aorta and aortic root aneurysm. Pre-operative transthoracic echocardiography gives information about the functions of heart valves. The prevention of hypertension during surgery will also prevent an undiagnosed ascending aorta aneurysm rupture or dissection. Therefore, the anesthesiologist must consider this condition and adjust the patient's hemodynamics and medication accordingly.

Declaration of interest: The authors report no conflicts of interest.

Financial Disclosure: No financial support was received.

\section{REFERENCES}

1. Ikizler M, Dernek S. A case of abdominal aortic aneurysm accompanied by horseshoe kidney. Turkish J Thorac Cardiovasc Surg 2005;13(4):381-383. 
2. Erdogan HB, Rabus MB, Omeroglu SN, Ogredik E, Goksedef D, Yakut C. Two-stage off-pump coronary artery bypass grafting and abdominal aortic aneurysm repair in a patient with horseshoe kidney. Turkish J Thorac Cardiovasc Surg 2005;13(3):245-248.

3. Stroosma OB, Kootstra G, Schurink GW. Management of aortic aneurysm in the presence of a horseshoe kidney. Br J Surg 2001;88:500-9. doi: 10.1046/j.13652168.2001.01718.x.

4. Artioukh DY, Wake PN, Edwards PR, Moody AP. Problems of abdominal aortic aneurysm associated with horseshoe kidney. Eur J Vasc Endovasc Surg 1997;14:758. doi: 10.1016/S1078-5884(97)80230-4.
5. Petrunic M, Stern-Padovan R, Mestrovic T. Abdominal and Iliac Arterial Aneurysms Associated with a Horseshoe Kidney. Should Aberrant Vascular Anatomy Mandate the Treatment Modality? EJVES Extra 2010;20:36-38. doi: 10.1016/j.ejvsextra.2010.08.002.

6. Yohannes P, Smith AD. The endourological management of complications associated with horseshoe kidney. J Urol 2002;168:5-8. doi: 10.1016/S0022-5347(05)64819-7. 